\title{
$\alpha$-adducin Gly460Trp polymorphism and essential hypertension risk in Chinese: a meta-analysis
}

\author{
Kuo Liu ${ }^{1,4}$, Ya Liu ${ }^{1,4}$, Jielin Liu1, Zuoguang Wang ${ }^{1}$, Yuqing Lou ${ }^{1}$, Yan Huang ${ }^{2}$, Qiuli Niu1, Wei Gu${ }^{1}$, \\ Xiaoling $\mathrm{Zhu}^{3}$ and Shaojun Wen ${ }^{1,5}$
}

No clear consensus has been reached on the $\alpha$-adducin polymorphism (Gly460Trp) and essential hypertension (EH) risk in Chinese. We conducted a meta-analysis in an effort to systematically explore the possible association. Case-control studies in Chinese and English performed with human subjects were identified by searching the MEDLINE, EMBASE, China Biological Medicine Database, China National Knowledge Infrastructure platform, Wanfang and VIP databases. The fixed-effects model and the random-effects model for dichotomous outcomes were applied to combine the results of the individual studies. We selected 20 studies that met the inclusion criteria, including a total of 5562 patients with hypertension and 4289 controls. Overall, our findings supported the hypothesis that the $A D D 1$ Gly460Trp polymorphism is associated with EH in the Chinese population. A borderline association was found between the tryptophan (Trp) allele of the Gly460Trp variant and hypertension $(P=0.05$, Odds ratio $(\mathrm{OR})=1.08,95 \%$ confidence interval $(\mathrm{Cl})=1.00-1.17$ and $P_{\text {heterogeneity }}=0.02$ ). Significantly increased risk was observed in the recessive genetic model $\left(P=0.0009,0 R=1.24,95 \% \mathrm{Cl}=1.09-1.41\right.$ and $\left.P_{\text {heterogeneity }}=0.04\right)$ as well as in the homozygote comparison $\left(P=0.006, \mathrm{OR}=1.25,95 \% \mathrm{Cl}=1.07-1.46\right.$ and $\left.P_{\text {heterogeneity }}=0.03\right)$. Furthermore, in the subgroup analysis, our results support a positive association among Chinese Han individuals $(P=0.001,0 R=1.25,95 \% \mathrm{Cl}=1.09-1.42$, $P_{\text {heterogeneity }}=0.08$, recessive genetic model; $P=0.009,0 R=1.26,95 \% \mathrm{Cl}=1.06-1.50, P_{\text {heterogeneity }}=0.03$, homozygote comparison). No apparent association was identified in Kazakhs. Our meta-analysis suggests that the Gly460Trp polymorphism might increase the risk of hypertension in Chinese populations, especially in Han Chinese. Further studies investigating gene-gene, gene-environment and mutual interactions are needed to better understand the role of $A D D 1$ in hypertension. Hypertension Research (2011) 34, 389-399; doi:10.1038/hr.2010.252; published online 13 January 2011

Keywords: $\alpha$-adducin; Chinese; meta-analysis; polymorphism

\section{INTRODUCTION}

Essential hypertension (EH) is a major public health problem that affects many members of developed countries as well as developing countries. According to the Nutrition and Health Survey of the People's Republic of China, the prevalence of hypertension was estimated to be $\sim 18.8 \%$ in the Chinese adult population, and 0.17 billion people were affected by it in 2002 (see ref. 1). This complex disorder is regarded as a multifactorial condition in which various hereditary and environmental factors have a role in determining the occurrence and development of the disease. Some research has suggested that interindividual variation in the risk of hypertension has a genetic component. $^{2}$ Approximately $20-60 \%$ of the population variability in blood pressure appears to be genetically determined. ${ }^{3}$ As a result, candidate genes for hypertension have been extensively studied.

Adducin is a ubiquitously expressed heterodimeric cytoskeleton protein composed of two subunits (an $\alpha$-subunit and either a $\beta$ - or $\gamma$-subunit depending on the tissue) that are encoded by three genes ( $A D D 1, A D D 2$ and $A D D 3$, respectively) located on different chromosomes. ${ }^{4}$ The human $\alpha$-adducin gene was mapped to chromosome 4 p16.3 and comprises 16 exons. Animal and clinical studies have suggested that an alteration in $A D D 1$ might be a contributing factor in the pathogenesis of hypertension. Previous studies using the Milan hypertensive rat strain model of hypertension showed that the $\alpha$-adducin gene point mutation could affect renal sodium transport

\footnotetext{
1Department of Hypertension Research, Beijing Anzhen Hospital, Capital Medical University and Beijing Institute of Heart Lung and Blood Vessel Diseases, Beijing, PR China; ${ }^{2}$ State Key Laboratory of Genetic Engineering, Institute of Genetics, School of Life Sciences, Fudan University, Shanghai, PR China; ${ }^{3}$ Emergency Center of Heart, Lung and Blood Vessel Diseases, Beijing Anzhen Hospital, Capital Medical University, Beijing, PR China

${ }^{4}$ These authors contributed equally to this work.

${ }^{5}$ Member of the International Society of Hypertension.

Correspondence: Dr X Zhu, Emergency Center of Heart, Lung and Blood Vessel Diseases, Beijing Anzhen Hospital, Capital Medical University, 2 Anzhen Road, Beijing 100029, PR China

E-mail: az9595@126.com.cn

or Dr S Wen, Department of Hypertension Research, Beijing Anzhen Hospital, Capital Medical University and Beijing Institute of Heart, Lung and Blood Vessel Diseases, 2 Anzhen Road, Beijing 100029, PR China.

E-mail: wenshaojun@yahoo.com.cn

Received 11 June 2010; revised 23 September 2010; accepted 2 October 2010; published online 13 January 2011
} 
and thus cause hypertension. ${ }^{5}$ The role of the $\alpha$-adducin gene in hypertension and other cardiovascular diseases has been extensively evaluated at the molecular level, with particular attention to the rs4961 (Gly460Trp, G460W or G460T) single nucleotide polymorphism at exon 10, (see ref. 6) where a guanine-to-thymine transversion at nucleotide 614 leads to a glycine (Gly) to tryptophan (Trp) substitution at amino acid position 460. In the last decade, a large number of studies seeking to link the Gly460Trp polymorphism to hypertension have been conducted in different races and ethnic groups, but the results have often been unreproducible. ${ }^{7-9}$ A similar situation also occurred in China. We therefore performed a formal meta-analysis to further evaluate the relationship of this polymorphism with $\mathrm{EH}$ in the Chinese population.

\section{METHODS}

\section{Identification and eligibility of relevant studies}

Genetic association studies evaluating the $\alpha$-adducin gene Gly460Trp polymorphism and EH in Chinese populations published before 1 May 2010 were included in our meta-analysis. We conducted a systematic computerized literature search of the PubMed, EMBASE, China Biological Medicine Database (http://cbm.imicams.ac.cn), China National Knowledge Infrastructure platform (http://www.cnki.net), Wanfang (http://www.wanfangdata.com.cn) and VIP (http://www.cqvip.com.cn) databases using the MeSH term 'Chinese' or 'China' in combination with the following keywords and MeSH terms: 'hypertension', ' $\alpha$-adducin' or 'ADD1', and 'polymorphism'. Search results were limited to studies on human subjects and articles in Chinese or English. The full text of the retrieved studies was scrutinized, and all of the references cited in the articles were searched to identify potentially relevant studies. To avoid data duplication when potentially overlapping populations were described in multiple reports, we selected the study that provided the fullest amount of information. Thus, individuals enrolled in more than one article were counted only once. If detailed genotyping information was not reported, we contacted the original authors to obtain the relevant data. All retrieved articles were screened to meet all the following criteria: (a) studies investigating the relationship between the ADD1 Gly460Trp polymorphism and hypertension in Chinese individuals; (b) use of an unrelated case-control design (familybased study designs with linkage considerations were excluded); (c) availability of genotype frequencies in both cases and controls; and (d) a genotype distribution of the control population consistent with Hardy-Weinberg equilibrium (HWE). Hypertension was defined as systolic blood pressure $\geqslant 140 \mathrm{~mm} \mathrm{Hg}$ and/or diastolic blood pressure $\geqslant 90 \mathrm{~mm} \mathrm{Hg}$ and/or treatment with anti-hypertensive medication. ${ }^{10,11}$

\section{Data extraction}

Two investigators (KL and YL) independently reviewed and extracted the data. Inconsistencies were discussed between the two investigators until a consensus was obtained on all of the items. If they could not reach an agreement, a third investigator (JL) adjudicated the disagreements. For each study, information was collected concerning the following characteristics: first author, year of publication, racial background and location of the study population, source of subjects, methodology used for genotype detection, diagnostic standard for hypertension, matching, clinical features, quality score, sample sizes, and distribution of genotypes and alleles in both case and control groups.

\section{Quality score assessment}

The quality assessment score we used was adapted from Niu et al. ${ }^{12}$ and Zhang et al., ${ }^{13}$ which were based on both traditional epidemiologic considerations and genetic issues raised by Thakkinstian et al. ${ }^{14,15}$ Total scores ranged from 0 (lowest) to 13 (highest). The criteria used for the quality assessment of the genetic association between the ADD1 Gly460Trp polymorphism and hypertension risk are summarized in the Appendix.

\section{Statistical analysis}

Odds ratios (ORs) with $95 \%$ confidence intervals (CIs) were used as the metric of choice. We calculated the OR and respective $95 \%$ CIs for each study to assess the strength of the association between the $\alpha$-adducin Gly460Trp polymorphism and the risk of $\mathrm{EH}$, according to the method described by Woolf. ${ }^{16}$ On the basis of the individual OR, the pooled ORs were assessed for allele comparison (Trp vs. Gly), dominant genetic model (GlyTrp+TrpTrp vs. GlyGly), recessive genetic model (TrpTrp vs. GlyTrp+GlyGly) and homozygote comparison (TrpTrp vs. GlyGly). The statistical significance was determined by the Z-test, and a $P$ value of $<0.05$ was considered statistically significant. In our study, two models of meta-analysis were used for dichotomous outcomes in the Review-Manager 4.2 software (Oxford, England, UK): the fixed-effects model and the random-effects model. The fixed-effects model used the Peto MantelHaenszel's method, which assumes that studies are sampled from populations with the same effect size, and adjusts the study weights according to the instudy variance. The random-effects model used the Der Simonian and Laird's method, which assumes that the studies are taken from populations with varying effect sizes, and calculates the study weights both from in-study and between-study variances, considering the extent of variation or heterogeneity. The chi-squared-based Q-test was performed to check the heterogeneity. ${ }^{17}$ A $P$ value $>0.10$ for the Q-test indicates a lack of heterogeneity among studies, and the pooled OR estimate of each study was calculated with the fixed-effects model. ${ }^{18}$ Otherwise, the random-effects model was used. ${ }^{18}$ The inconsistency index $I^{2}$ was also calculated to quantify the degree of heterogeneity between studies, with $I^{2}<25 \%, 25-50 \%, 50-75 \%$ and $>75 \%$ to represent low, moderate, large and extreme heterogeneity, respectively. ${ }^{19}$ For each genetic contrast, subgroup analysis according to racial descent was only performed for Han Chinese and non-Han Chinese minorities to estimate ethnic-specific ORs when there were at least three independent studies. We also classified all the Han populations as either Northern Han Chinese (N-Han) or Southern Han Chinese (S-Han), with the Yangtze River serving as a geographical boundary. ${ }^{20-22}$ Additionally, the source of heterogeneity was further investigated with stratified meta-analyses based on sample sources (population-based or hospital-based).

Sensitivity analyzes were conducted by sequentially deleting each individual study in an attempt to identify the potential influence of each individual data set to the pooled ORs. In addition, an estimate of potential publication bias was carried out by the funnel plot, in which the standard error of $\log (\mathrm{OR})$ of each study was plotted against its OR. The symmetry of the plot distribution indicates the absence of publication bias. Funnel-plot asymmetry was assessed with Egger's linear regression test. ${ }^{23}$ The significance of the intercept was determined by the $t$-test suggested by Egger, and $P<0.05$ was considered representative of statistically significant publication bias. HWE was tested with a chi-squared test for goodness of fit based on a Web program (http:// www.ihg.gsf.de/cgi-bin/hw/hwal.pl).

All statistical analyses were performed using the software Review-Manager 4.2 and Stata version 10.0 (Stata Corporation, College Station, TX, USA). All statistical tests were two-sided.

\section{RESULTS}

\section{Description of studies identified in meta-analysis}

Through a comprehensive search, 29 potentially relevant articles concerning the $\alpha$-adducin gene Gly460Trp polymorphism and hypertension in the Chinese population were identified on the basis of our inclusion criteria. A total of 8 of the 29 eligible articles were excluded because they shared the same or overlapping data. We also deleted one study $^{24}$ in which the genotype distributions in control individuals departed from HWE $\left(\mathrm{P}_{\mathrm{HWE}}=0.00466\right)$. Finally, a total of 20 studies containing 5562 hypertensive patients and 4289 controls were used in this meta-analysis. ${ }^{25-44}$ The study by Zhan et al. ${ }^{35}$ was an unpublished thesis obtained from a medical doctorate dissertation database that was a sub-database of the CNKI and Wanfang databases. Three studies (Jiang et al., ${ }^{26}$ Zhao et al., ${ }^{31}$ and Huang et al. ${ }^{36}$ ) included in our metaanalysis only listed the percentages of genotype frequencies in both cases and controls. We therefore calculated the numbers of the three different genotypes (GlyGly, GlyTrp and TrpTrp). To avoid possible bias by calculating, we contacted the original or corresponding authors by e-mail to verify the data. All of them replied and offered the 


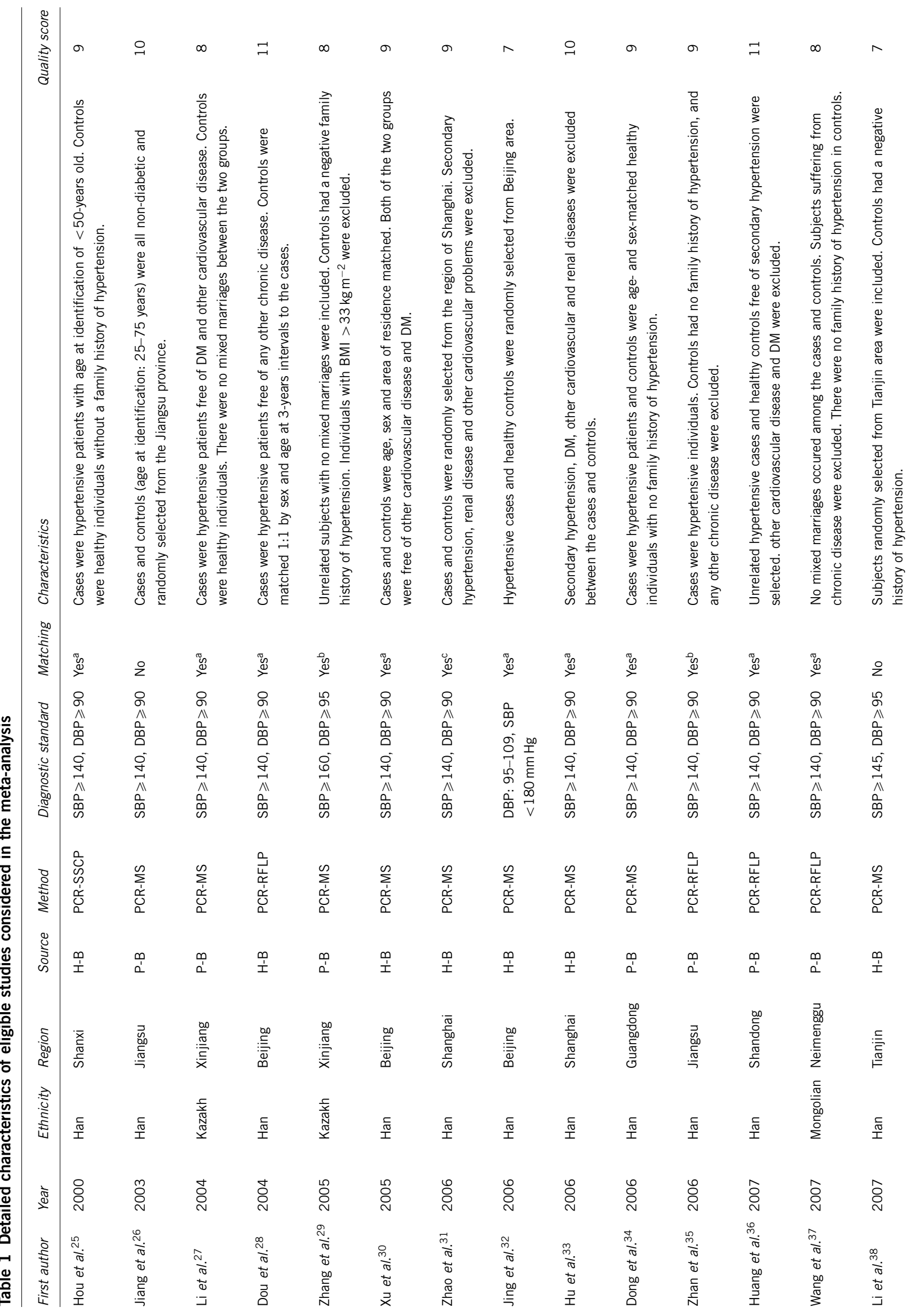


accurate data. The results of our quality score assessment varied between 7 and 11, indicating that most studies identified were of medium or high quality.

Of the 20 studies, 12 were hospital-based and 8 were populationbased studies. The populations of these studies were as follows: 16 studies included Han subjects (4826 cases and 3777 controls), 3 included Kazakh subjects (636 cases and 462 controls) and 1 included Mongolian subjects (100 cases and 50 controls). The latter two ethnicities were Northern Chinese minorities. In total, $65 \%$ of the studies (13/20) stated that age and gender were well-matched between the case and control groups, $75 \%$ (15/20) were age-matched and $80 \%$ $(16 / 20)$ were gender-matched. All studies used blood samples for genotyping. The detailed characteristics of all eligible studies in the present meta-analysis are described in Table 1, including the first author, year of publication, ethnicity, population location, source of subjects, genotyping method, diagnostic standard for hypertension, matching, main information extracted from each study and quality score. Sample sizes, genotype numbers, allele frequency in both cases and controls, and $P$ values of HWE in controls are listed in Table 2.

\section{Main meta results}

In the meta-analysis for all subjects, the pooled overall frequency of the Trp allele was $49.96 \%$ for hypertensive cases and $47.74 \%$ for normotensive controls. For each study, we investigated the association between the $\alpha$-adducin gene Gly460Trp polymorphism and EH risk, assuming different inheritance models of the 460Trp allele. The main results of this meta-analysis and the heterogeneity test are shown in Table 3. The random-effects model was used to pool the result, as the between-study heterogeneity was significant. Overall, a marginal significantly increased risk was found in the allele comparison (Trp vs. Gly: $P=0.05, \mathrm{OR}=1.08,95 \% \mathrm{CI}=1.00-1.17, P_{\text {heterogeneity }}=0.02$ and $I^{2}=42.3 \%$ ) (Figure 1a), and a significant association was observed in the recessive genetic model (TrpTrp vs. GlyTrp+GlyGly: $P=0.0009$, $\mathrm{OR}=1.24,95 \% \mathrm{CI}=1.09-1.41, \quad P_{\text {heterogeneity }}=0.04$ and $I^{2}=38.7 \%$ ) (Figure 1b), as well as in the homozygote comparison (TrpTrp vs. GlyGly: $P=0.006, \mathrm{OR}=1.25,95 \% \mathrm{CI}=1.07-1.46, P_{\text {heterogeneity }}=0.03$ and $I^{2}=41.2 \%$ ) (Figure 1c). However, we found no positive association in the dominant genetic model (GlyTrp+TrpTrp vs. GlyGly: $P=0.22, \quad \mathrm{OR}=1.09, \quad 95 \% \quad \mathrm{CI}=0.95-1.24, \quad P_{\text {heterogeneity }}=0.01 \quad$ and $I^{2}=46.5 \%$ ) (Table 3).

\section{Subgroup analyses}

In the subgroup analysis by ethnicity, the studies included were stratified as Han Chinese and Chinese Kazakhs, except for the Chinese Mongolians, among whom only one study with less data was performed. The Trp allele had a similar representation in cases and controls of Han Chinese (49.94 and 47.75\%, respectively) and Chinese Kazakhs (48.74 and 47.94\%, respectively). Although moderate heterogeneity existed among Han Chinese in all genetic models, significant association was maintained in some subgroup analyses, especially in the recessive genetic model (TrpTrp vs. GlyTrp+GlyGly: $P=0.001$, $\mathrm{OR}=1.25,95 \% \mathrm{CI}=1.09-1.42, \quad P_{\text {heterogeneity }}=0.08$ and $I^{2}=35.3 \%$ ) (Figure $2 \mathrm{a}$ ) and in the homozygote comparison (TrpTrp vs. GlyGly: $P=0.009, \quad \mathrm{OR}=1.26, \quad 95 \% \quad \mathrm{CI}=1.06-1.50, \quad P_{\text {heterogeneity }}=0.03$ and $I^{2}=44.7 \%$ ) (Figure $2 \mathrm{~b}$ ). When the subgroup analysis was limited to Chinese Kazakhs, no heterogeneity was detected in any genetic model. No evidence for an association between the Gly460Trp polymorphism and hypertension risk was obtained (Table 3).

Furthermore, we also divided the Han Chinese into two subgroups: $\mathrm{N}-\mathrm{Han}$ and S-Han. The fixed-effects model was used to pool 
Table 2 Sample size of each study, the distribution of Gly460Trp genotypes and T allele frequencies of cases and controls and $P$-values of HWE in controls

\begin{tabular}{|c|c|c|c|c|c|c|c|c|c|c|c|}
\hline \multirow[b]{2}{*}{ First author } & \multicolumn{2}{|c|}{ Sample size } & \multicolumn{2}{|c|}{ GG genotype } & \multicolumn{2}{|c|}{ GT genotype } & \multicolumn{2}{|c|}{ TT genotype } & \multicolumn{2}{|c|}{ Tallele frequency (\%) } & \multirow{2}{*}{$\frac{\text { HWE } P^{a} \text { value }}{\text { Controls }}$} \\
\hline & Cases & Controls & Cases & Controls & Cases & Controls & Cases & Controls & Cases & Controls & \\
\hline Hou et al. ${ }^{25}$ & 183 & 129 & 54 & 36 & 84 & 66 & 45 & 27 & 47.54 & 46.51 & 0.75 \\
\hline Jiang et al. ${ }^{26}$ & 189 & 147 & 44 & 35 & 94 & 79 & 51 & 33 & 51.85 & 49.32 & 0.36 \\
\hline Li et al. ${ }^{27}$ & 235 & 132 & 56 & 35 & 119 & 62 & 60 & 35 & 50.85 & 50.00 & 0.49 \\
\hline Dou et al. ${ }^{28}$ & 234 & 234 & 42 & 66 & 113 & 117 & 79 & 51 & 42.09 & 46.79 & 0.95 \\
\hline Zhang et al. ${ }^{29}$ & 278 & 220 & 71 & 58 & 144 & 121 & 63 & 41 & 48.56 & 46.14 & 0.11 \\
\hline Xu et al. ${ }^{30}$ & 348 & 184 & 91 & 49 & 174 & 88 & 83 & 47 & 48.85 & 49.46 & 0.56 \\
\hline Zhao et al. ${ }^{31}$ & 278 & 231 & 117 & 64 & 72 & 112 & 89 & 55 & 44.96 & 48.05 & 0.66 \\
\hline Jing et $a l^{32}$ & 463 & 260 & 110 & 70 & 262 & 136 & 91 & 54 & 47.95 & 46.92 & 0.42 \\
\hline Hu et $a .^{33}$ & 396 & 214 & 114 & 59 & 200 & 104 & 82 & 51 & 45.96 & 48.13 & 0.70 \\
\hline Dong et al. ${ }^{34}$ & 97 & 87 & 23 & 21 & 49 & 40 & 25 & 26 & 51.03 & 52.87 & 0.47 \\
\hline Zhan et al. ${ }^{35}$ & 190 & 94 & 43 & 17 & 98 & 53 & 49 & 24 & 51.58 & 53.72 & 0.19 \\
\hline Huang et al. ${ }^{36}$ & 256 & 495 & 59 & 124 & 107 & 237 & 90 & 134 & 56.05 & 51.01 & 0.35 \\
\hline Wang et al.. ${ }^{37}$ & 100 & 50 & 20 & 12 & 43 & 31 & 37 & 7 & 58.50 & 45.00 & 0.07 \\
\hline Li et al. ${ }^{38}$ & 80 & 80 & 17 & 25 & 42 & 38 & 21 & 17 & 52.50 & 45.00 & 0.72 \\
\hline Bian et al. ${ }^{39}$ & 160 & 151 & 35 & 40 & 79 & 69 & 46 & 42 & 53.44 & 50.66 & 0.29 \\
\hline Huang et al. ${ }^{40}$ & 123 & 110 & 42 & 31 & 51 & 50 & 30 & 29 & 45.12 & 49.09 & 0.34 \\
\hline Lu et al. ${ }^{41}$ & 200 & 200 & 50 & 73 & 102 & 96 & 48 & 31 & 49.50 & 39.50 & 0.95 \\
\hline Gong et al. ${ }^{42}$ & 196 & 192 & 38 & 42 & 84 & 101 & 74 & 49 & 59.18 & 51.82 & 0.46 \\
\hline Lin et al. ${ }^{43}$ & 1081 & 604 & 311 & 215 & 540 & 298 & 230 & 91 & 46.25 & 39.74 & 0.46 \\
\hline Niu et al. ${ }^{44}$ & 475 & 475 & 110 & 105 & 216 & 231 & 149 & 139 & 54.11 & 53.58 & 0.63 \\
\hline Total & 5562 & 4289 & 1447 & 1177 & 2673 & 2129 & 1442 & 983 & 49.96 & 47.74 & 0.73 \\
\hline
\end{tabular}

Abbreviation: HWE, Hardy-Weinberg equilibrium.

aThe $P$-value of HWE determined by the $\chi^{2}$ test.

the results in N-Han, as the between-study heterogeneity was insignificant. The results revealed significant associations for hypertension risk with the Gly460Trp polymorphism in the dominant genetic model (GlyTrp+TrpTrp vs. GlyGly: $P=0.02$, $\mathrm{OR}=1.21,95 \%$ $\mathrm{CI}=1.03-1.41, P_{\text {heterogeneity }}=0.54$ and $I^{2}=0 \%$ ), the recessive genetic model (TrpTrp vs. GlyTrp+GlyGly: $P=0.002, \quad \mathrm{OR}=1.28, \quad 95 \%$ $\mathrm{CI}=1.10-1.49, P_{\text {heterogeneity }}=0.12$ and $\left.I^{2}=39.6 \%\right)$ and the homozygote comparison (TrpTrp vs. GlyGly: $P=0.001, \mathrm{OR}=1.36,95 \% \mathrm{CI}=1.13$ $1.64, P_{\text {heterogeneity }}=0.24$ and $I^{2}=23.9 \%$ ) (Table 3). In S-Han, notable heterogeneity was discerned in all genetic models except for the recessive genetic model, in which a weak association was found (TrpTrp vs. GlyTrp+GlyGly: $P=0.002, \mathrm{OR}=1.24,95 \% \mathrm{CI}=1.08-1.42$, $P_{\text {heterogeneity }}=0.12$ and $I^{2}=39.0 \%$ ) (Table 3 ).

In the analyses stratified by sample sources, significant associations were found both in population-based (TrpTrp vs. GlyTrp+GlyGly: $P=0.02, \quad \mathrm{OR}=1.23, \quad 95 \% \quad \mathrm{CI}=1.03-1.47, \quad P_{\text {heterogeneity }}=0.15 \quad$ and $\left.I^{2}=35.4 \%\right)$ and in hospital-based (TrpTrp vs. GlyTrp+GlyGly: $P=0.005, \quad \mathrm{OR}=1.26, \quad 95 \% \quad \mathrm{CI}=1.07-1.48, \quad P_{\text {heterogeneity }}=0.04$ and $I^{2}=45.1 \%$ ) subgroups under the recessive model (Table 3 ). In the homozygote comparison, significantly elevated risk was found in hospital-based studies (TrpTrp vs. GlyGly: $P=0.02$, $O R=1.30,95 \%$ $\mathrm{CI}=1.05-1.61, P_{\text {heterogeneity }}=0.01$ and $I^{2}=55.2 \%$ ) (Table 3). Moreover, to uncover the potential influence of the studies whose diagnostic standards differed from others (Zhang et al., ${ }^{29}$ Jing et al. ${ }^{32}$ and Li et $a l^{38}$ ), repeated analyses have been conducted by omitting these three studies. Consistent with our previous findings, similar results were obtained when deleting the three studies. Significant associations were observed among the overall population, Han Chinese, N-Han and S-Han, and again, no positive association was found in Chinese Kazaks (data not shown).

\section{Sensitivity analysis}

Sensitivity analyses were conducted to assess whether each individual study affected the final results. Our analysis indicated that three independent studies by Dou et al., ${ }^{28}$ Zhao et al. ${ }^{31}$ and Lin et al. ${ }^{43}$ were the main cause of heterogeneity across all subjects and Han Chinese. The heterogeneity was effectively removed after the exclusion of these three studies (Dou et al.: ${ }^{28} P_{\text {heterogeneity }}=0.10, I^{2}=30.7 \%$, homozygote comparison; Zhao et al. ${ }^{31} P_{\text {heterogeneity }}=0.36, I^{2}=7.9 \%$, dominant genetic model; and Lin et al.: ${ }^{43} P_{\text {heterogeneity }}=0.10$, $I^{2}=30.5 \%$, allele comparison) for total comparisons. Data for Han Chinese are not shown. In addition, sensitivity analyses suggested that the corresponding summary ORs were substantially altered among Han Chinese when excluding these three studies (data not shown). There was no single study that influenced the pooled OR qualitatively in the subgroup of Chinese Kazakhs.

\section{Publication bias}

Begg's funnel plot and Egger's test were used to evaluate the literature publication bias for allele comparison (Trp vs. Gly). The shape of the funnel plot did not reveal any evidence of obvious asymmetry (Figure 3), and Egger's test suggested the absence of publication bias among all the studies included $(t=-0.56, P=0.581)$.

\section{DISCUSSION}

We performed a systematic review of the literature by means of a meta-analysis on the association between the $\alpha$-adducin Gly460Trp polymorphism and $\mathrm{EH}$, without evidence of publication bias for the outcome. On the basis of 5562 essential hypertensive individuals and 4289 controls in total, we concluded that the $\alpha$-adducin gene Gly460Trp polymorphism appeared to be associated with an increased 
Table 3 Summary ORs and $95 \% \mathrm{Cl}$ of Gly460Trp polymorphism and hypertension risk under various genetic contrasts

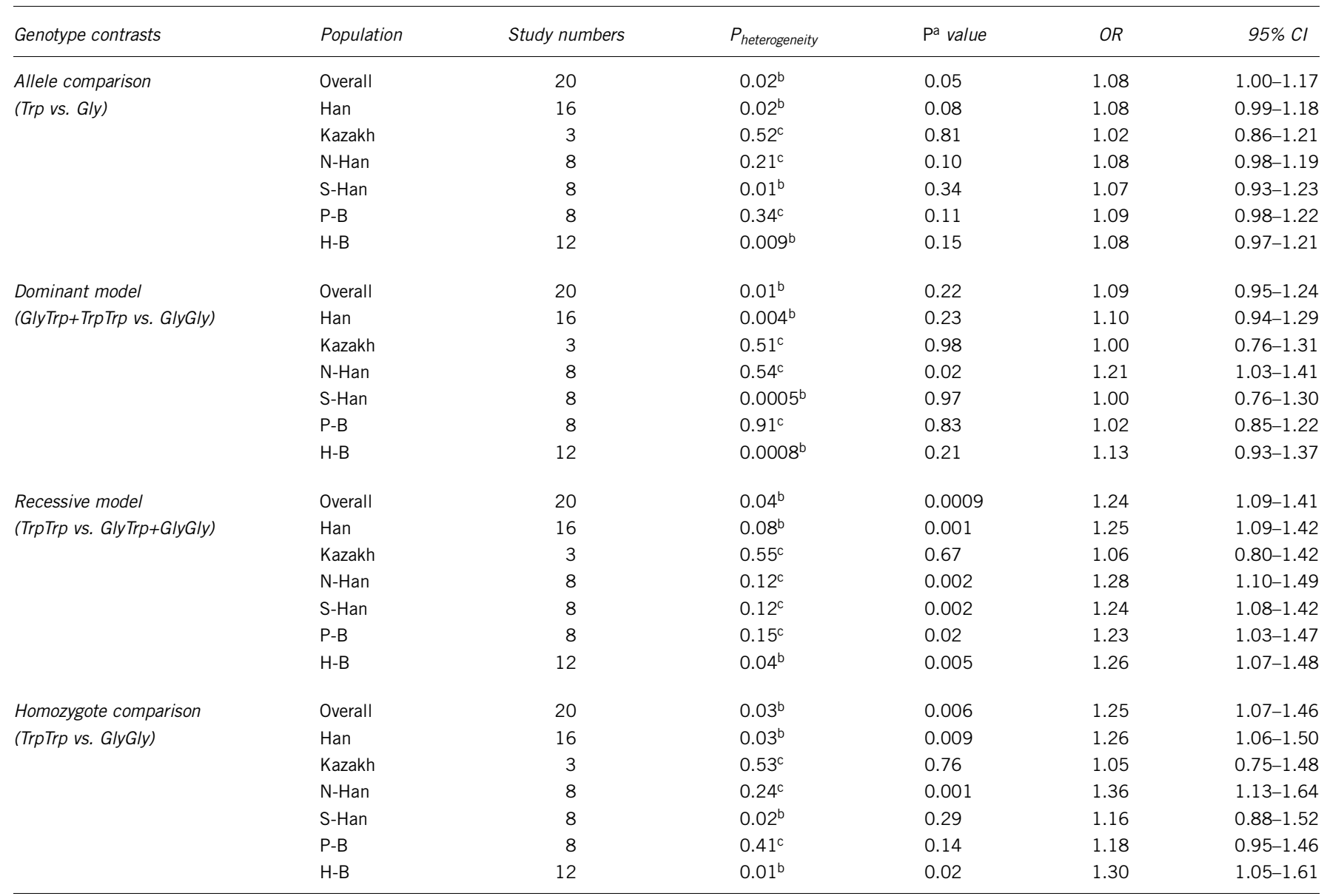

Abbreviations: OR, odds ratio; Cl, confidence interval; N-Han, northern Han Chinese; S-Han, southern Han Chinese; P-B, population-based study; H-B, hospital-based study.

aThe $P$-value of $\mathrm{OR}$ determined by the $\mathrm{Z}$ test.

bRandom effect estimate.

'Fixed effect estimate.

risk of hypertension in Chinese populations, especially in Han Chinese.

In 1997, Cusi et al. ${ }^{7}$ first reported that the 460Trp allele of $\alpha$-adducin was associated with hypertension in human subjects, particularly in a salt-sensitive form of EH. Subsequently, Iwai et al. ${ }^{45}$ found that the Gly460Trp polymorphism seemed to be involved in the pathophysiology of hypertension among Japanese subjects. Nevertheless, some studies have shown inconsistent results as well as strong racial and regional disparities. A recent case-control study and metaanalysis by Niu et al. ${ }^{44}$ failed to demonstrate the genetic association of the ADD1 gene Gly460Trp polymorphism with hypertension. There are many possible reasons for this variability, and ethnic specificity and population structure may be the most important potential confounding factors. It is necessary to reconcile the conflicting findings in a genetically well-defined population. Our meta-analysis was focused on a single ethnicity or country, and accordingly eliminated racial differences. The results indicated that there was a significant association with increased risk among Chinese hypertensive individuals.

The molecular mechanism hypothesized for the Gly460Trp polymorphism is likely associated with a salt-sensitive form of hypertension. A previous study demonstrated that subjects bearing one Trp allele in $\alpha$-adducin displayed increased renal tubular sodium reabsorption and retention as a result of a reduced renal pressure-natriuresis slope after sodium depletion or sodium load. ${ }^{46}$ Further studies revealed similar results and demonstrated that the genetic variant modulated sodium transport through variations in sodium-potassium ATPase activity on the basolateral membrane and through modifications in the assembly of the actin cytoskeleton. ${ }^{47}$ A systematic review by Beeks et al. ${ }^{48}$ summarized seven studies addressing the association between the $\alpha$-adducin Gly460Trp polymorphism and salt sensitivity. Five of these seven studies were performed by the same research group and showed evidence to support the significant association, $, 46,49-51$ but one Polish study failed to obtain the same conclusion. ${ }^{52}$ Another article by Grant et al. ${ }^{53}$ reported that the systolic blood pressure response to sodium changes was significantly greater in subjects with homozygous 460Trp compared with subjects carrying other genotypes. In addition, a newly published meta-analysis investigating the association between the ADD1 polymorphism (Gly460Trp) and the genetic predisposition to salt sensitivity confirmed that although the overall results showed no increased risk of salt sensitivity for adducin Trp allele carriers $(P=0.08, \mathrm{OR}=1.40$ and $95 \% \mathrm{CI}=0.96-2.04)$, they did show that the association between Gly460Trp and salt sensitivity is statistically significant in a subgroup of Asian people $(P=0.02$, $\mathrm{OR}=1.33$ and $95 \% \mathrm{CI}=1.06-1.69$ ) (see ref. 54). This is consistent with our results. To summarize, it appears that the $\alpha$-adducin gene mutation might affect overall body sodium metabolism and therefore trigger hypertension. 


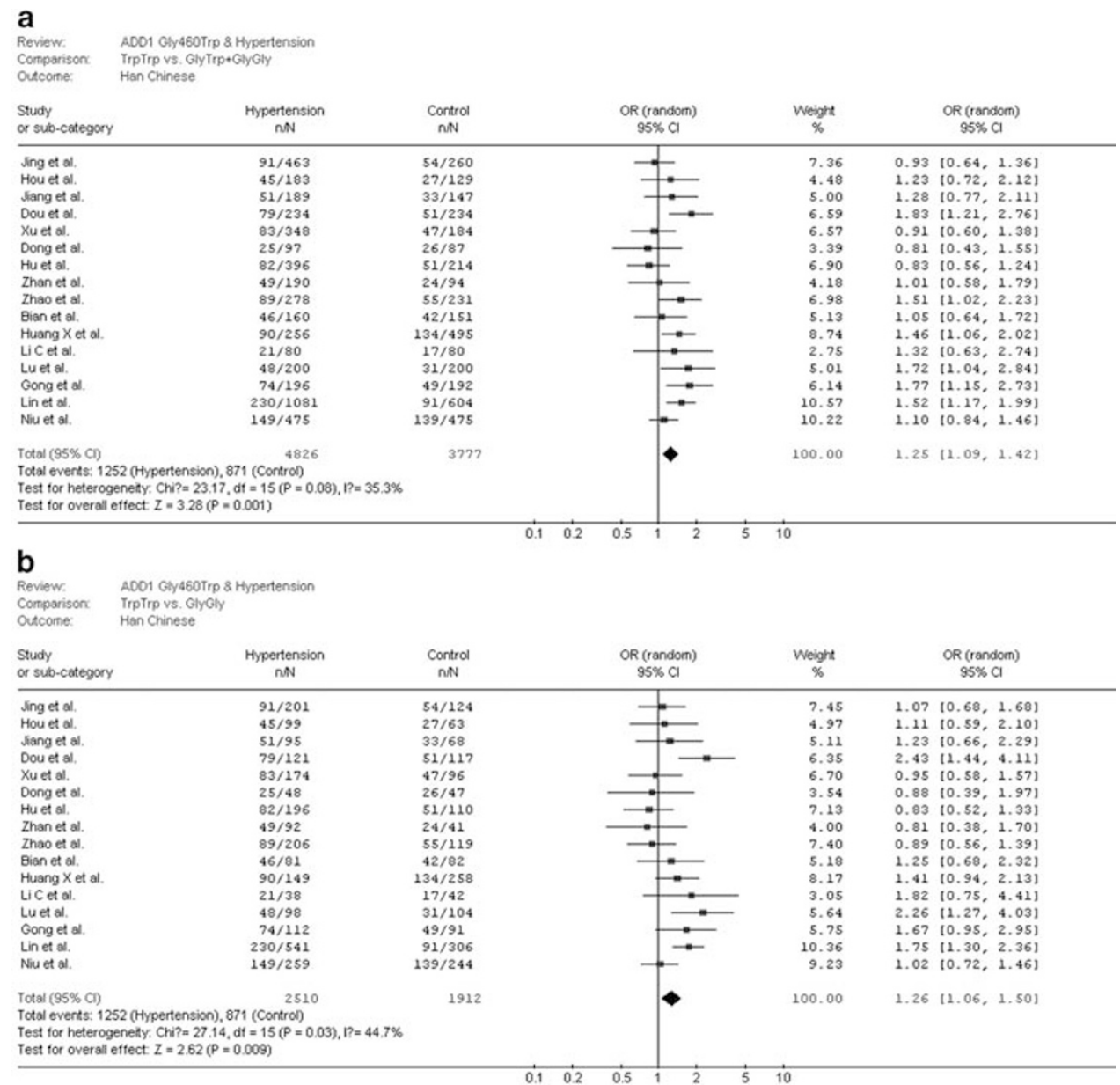

Figure 2 (a) Meta-analysis for the association between Gly460Trp polymorphism and hypertension under a recessive genetic model (TrpTrp vs. GlyTrp+GlyGly) in Han Chinese individuals using a random-effects model. $\mathrm{N}$ indicates the total number of Han Chinese individuals and $\mathrm{n}$ indicates the total number of TrpTrp. (b) Meta-analysis for the association between Gly460Trp polymorphism and hypertension in the contrast of TrpTrp vs. GlyGly in Han Chinese individuals using a random-effects model. $\mathrm{N}$ indicates the total number of TrpTrp plus GlyGly and $\mathrm{n}$ indicates the total number of TrpTrp.

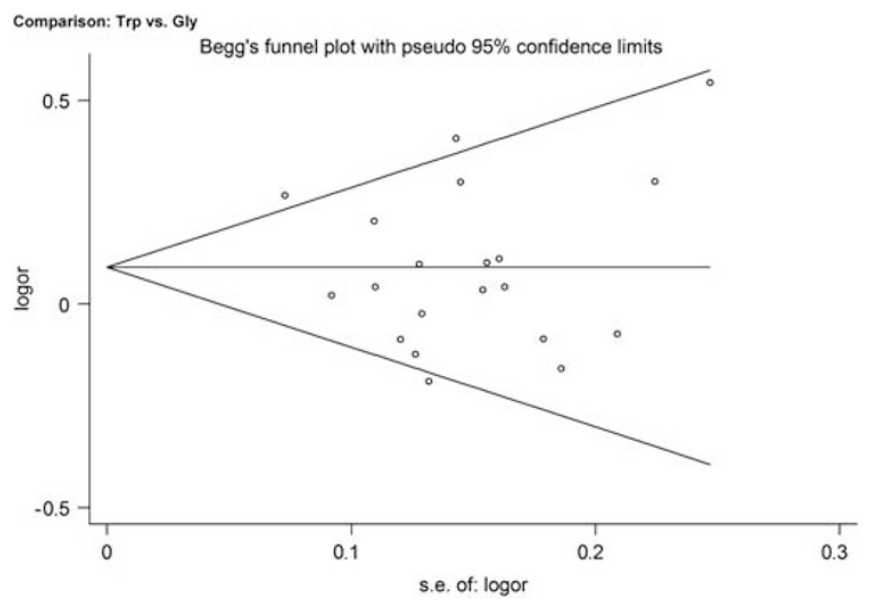

Figure 3 Begg's funnel plot of Egger's test of the Gly460Trp polymorphism allele comparison (Trp vs. Gly) for publication bias. No asymmetry was found, as indicated by the $P$-value of Egger's test.

China is the most populous country in the world, and the Han are the largest ethnicity. In the subgroup analyses, we divided all studies into two subgroups: Han Chinese and Chinese Kazakhs. Statistically significant associations were found among studies of Han Chinese.
In the Han population, the individuals were further distinguished as $\mathrm{N}-\mathrm{Han}$ and S-Han because they have different genetic backgrounds. This has been verified by a series of analyses of genetic markers, ${ }^{21,55-57}$ anthropological data concerning somatometric and nonmetric features $^{58,59}$ and dermatoglyphic parameters. ${ }^{22,60}$ In addition, environmental factors, such as geographical location, climate, eating habits and lifestyle all contribute to the North-South differences. In our current study, the Trp allele frequencies in the N-Han population (52.16\% in cases and $49.10 \%$ in controls) were higher than those in the S-Han population ( $48.47 \%$ in cases and $46.61 \%$ in controls). The North-South differences were proven statistically significant using the chi-squared test $\left(\chi^{2}=12.613, P<0.0001\right.$ for cases and $\chi^{2}=4.652$, $P=0.031$ for controls, respectively). When analyzing the gene-disease interaction, our research findings suggested that the $\alpha$-adducin gene Gly460Trp polymorphism was associated with $\mathrm{EH}$ in both the N-Han and S-Han. Compared with the Han population, no association was detected under any genetic models of Chinese Kazakhs. Previous research showed that the genetic backgrounds of these two ethnic groups are very different. The Kazakh ethnic group resulted from genetic mixing of Caucasians and East Asians. ${ }^{61-63}$ The genetic contribution of Caucasian in Chinese Kazakhs is 30.2\% (see ref. 62). Our previous report showed that there is no association between hypertension and the $\alpha$-adducin Gly460Trp polymorphism in the Caucasian population. ${ }^{64} \mathrm{~A}$ series of studies suggested that the 
McGregor K, Sechtem U, Silber S, Tendera M, Widimsky P, Zamorano JL, Erdine S, Kiowski W, Agabiti-Rosei E, Ambrosioni E, Lindholm LH, Viigimaa M, Adamopoulos S, Agabiti-Rosei E, Ambrosioni E, Bertomeu V, Clement D, Erdine S, Farsang C, Gaita D, Lip G, Mallion JM, Manolis AJ, Nilsson PM, O’Brien E, Ponikowski P, Redon J, Ruschitzka F, Tamargo J, van Zwieten P, Waeber B, Williams B. 2007 guidelines for the management of arterial hypertension: the task force for the management of arterial hypertension of the European Society of Hypertension (ESH) and of the European Society of Cardiology (ESC). J Hypertens 2007; 25: 1105-1187.

11 Ogihara T, Kikuchi K, Matsuoka H, Fujita T, Higaki J, Horiuchi M, Imai Y, Imaizumi T, Ito $\mathrm{S}$, Iwao $\mathrm{H}$, Kario K, Kawano $\mathrm{Y}$, Kim-Mitsuyama S, Kimura G, Matsubara H, Matsuura H, Naruse M, Saito I, Shimada K, Shimamoto K, Suzuki H, Takishita S, Tanahashi N, Tsuchihashi T, Uchiyama M, Ueda S, Ueshima H, Umemura S, Ishimitsu T, Rakugi H. The Japanese Society of Hypertension guidelines for the management of hypertension (JSH 2009). Hypertens Res 2009; 32: 3-107.

12 Niu W, Qi Y, Qian Y, Gao P, Zhu D. The relationship between apolipoprotein E epsilon2/ epsilon3/epsilon4 polymorphisms and hypertension: a meta-analysis of six studies comprising 1812 cases and 1762 controls. Hypertens Res 2009; 32: 1060-1066.

13 Zhang YG, Huang J, Zhang J, Li XB, He C, Xiao YL, Tian C, Wan H, Zhao YL, Tsewang YG, Fan H. RANTES gene polymorphisms and asthma risk: a meta-analysis. Arch Med Res 2010; 41: 50-58.

14 Thakkinstian A, McEvoy M, Minelli C, Gibson P, Hancox B, Duffy D, Thompson J, Hall I, Kaufman J, Leung TF, Helms PJ, Hakonarson H, Halpi E, Navon R, Attia J. Systematic review and meta-analysis of the association between $\beta 2$-adrenoceptor polymorphisms and asthma: a HuGE review. Am J Epidemiol 2005; 162: 201-211.

15 Attia J, Thakkinstian A, D'Este C. Meta-analyses of molecular association studies: methodologic lessons for genetic epidemiology. J Clin Epidemiol 2003; 56: 297-303.

16 Woolf B. On estimating the relation between blood group and disease. Ann Hum Genet 1955; 19: 251-253.

17 Lau J, Ioannidis JP, Schmid CH. Quantitative synthesis in systematic reviews. Ann Intern Med 1997; 127: 820-826.

18 Petitti DB. Meta-analysis, Decision Analysis, and Cost-effectiveness Analysis. Oxford University Press: NewYork, 1994.

19 Higgins JP, Thompson SG, Deeks JJ, Altman DG. Measuring inconsistency in meta-analyses. BMJ 2003; 327: 557-560.

20 Xiao C, Cavalli-Sforza LL, Minch E, Du R. Principal component analysis of gene frequencies of Chinese populations. Sci China C Life Sci 2000; 43: 472-481.

21 Wen B, Li H, Lu D, Song X, Zhang F, He Y, Li F, Gao Y, Mao X, Zhang L, Qian J, Tan J, Jin J, Huang W, Deka R, Su B, Chakraborty R, Jin L. Genetic evidence supports demic diffusion of Han culture. Nature 2004; 431: 302-305.

22 Zhang HG, Chen YF, Ding M, Jin L, Case DT, Jiao YP, Wang XP, Bai CX, Jin G, Yang JM, Wang H, Yuan JB, Huang W, Wang ZG, Chen RB. Dermatoglyphics from all Chinese ethnic groups reveal geographic patterning. PLoS One 2010; 5: e8783.

23 Egger M, Davey Smith G, Schneider M, Minder C. Bias in meta-analysis detected by a simple, graphical test. BMJ 1997; 315: 629-634.

24 Zhao H, Cao J, Zhou L, Wang B, Qiu C. Genetic analysis of $\alpha$-ADDUCIN and GNB3 in essential hypertension patient. Ji Chu Yi Xue Yu Lin Chuang 2009; 29: 393-396.

25 Hou R, Liu Z, Xue M, Wang Y, Ye T, Sun C, Wang Z, Liu Y. Is Gly460Trp variant of $\alpha$-adducin associated with essential hypertension in the Hans of Chinese population. Zhonghua Yi Xue Yi Chuan Xue Za Zhi 2000; 17: 413-416.

26 Jiang S, Li X, Zhang H, Wang Z, De W, Yuan S, Huang J. Polymorphism of alpha-adducin, angiotensin-converting enzyme (ACE) genes, environmental factors and essential hypertension. Zhonghua Gao Xue Ya Za Zhi 2003; 11: 563-566.

27 Li N, Li H, Zhou L, Nu E, Ouyang W, Bu K, Cheng Q, Wang Y, Ren R. The relationship between the $\alpha$-adducin gene variant and essential hypertension in Kazakans of Xinjiang. Ke Xue Ji Shu Yu Gong Cheng 2004; 4: 551-555.

28 Dou X, Sun K, Huang X, Liu X, Ju Z, Zhang H. Adducin and angiotensin converting enzyme polymorphisms in essential hypertension. Zhongguo Xun Huan Za Zhi 2004; 19: $286-289$.

29 Zhang Z, Liu Y, Lin R, Wang S, Wang X, Wen H, Jin L. Connection between ADD1 gene polymorphism and essential hypertension from Xinjiang Kazakhs. Fu Dan Xue Bao Yi Xue Ban 2005; 32: 239-242.

30 Xu J, Hua Q, Li D, Liu R, Yang Z, Chen H, Guo J. The association of gene polymorphism of $\alpha$-adducin gene Gly460Trp with essential hypertension in Han nationality patients in Beijing. Yi Xue Yan Jiu Sheng Xue Bao 2005; 18: 999-1004.

31 Zhao L, Gao P, Zhu D. Relationship between combination effect of ACE and adducin genes and essential hypertension. Zhongguo Xin Xue Guan Za Zhi 2006; 11: 16-19.

32 Jing S, Sun N, Wang H, Ma Z. Association of the $\alpha$-adducin Gly460Trp polymorphism with essential hypertension and blood pressure-lowing response to valsartan hydrochlorothiazide. Zhongguo Lin Chuang Yao Li Xue Za Zhi 2006; 22: 112-115.

$33 \mathrm{Hu} \mathrm{B}$, Chu S, Wang J, Wang G, Gao P, Zhu D. Single nucleotide polymorphisms of three candidate genes in essential hypertension. Zhonghua Ne Ke Za Zhi 2006; 45: 281-284.

34 Dong H, Li Q, Wang Q, Luo Z. Association analysis between genetic polymorphism of ADD1 gene and GNB3 gene and essential hypertension. Ling Nan Xin Xue Guan Bing Za Zhi 2006; 12: 258-261.

35 Zhan Y. Studies on Candidate Genes of Essential Hypertension and Antihypertensive Pharmacogenomics. Nan Jing Yi Ke Da Xue: Nanjing, 2006.

36 Huang $X$, Sun K, Song Y, Zhang H, Yang Y, Hui R. Association of $\alpha$-adducin gene and G-protein $\beta 3$-subuint gene with essential hypertension in Chinese. Zhonghua Yi Xue Za Zhi 2007; 87: 1682-1684.

37 Wang C, Sun G, Yan X, Ding Y. Study of $\alpha$-adducin and endothelial nitric oxide synthase gene polymorphism in patients with essential hypertension in mongulia population. Lin Chuang Xin Xue Guan Bing Za Zhi 2007; 23: 525-527.
38 Li C, Dang Q, Mu H. Association study between essential hypertension and $\alpha$-adducin P460Trp mutation. Guo Ji Xin Xue Guan Bing Za Zhi 2007; 34: 472-474.

39 Bian S, Geng $Q$, Yu M, Zhang Y, Xie Y. Relationship between $\alpha$-adducin gene polymorphism and essential hypertension. Lin Chuang Xin Xue Guan Bing Za Zhi 2007; 23: 897-900

40 Huang G, Wu P, Zhou T, Deng F. The relationship between $\alpha$-adducin gene mutation and essential hypertension in Hazakh people of Shihezi district in Xinjiang Uygur autonomous region. Shi He Zi Da Xue Xue Bao Zi Ran Ke Xue Ban 2008; 26: 12-15.

41 Lu L, Chen H, Yu L, Luo J, Wu X, Yang L. Association of $\alpha$-adducin and angiotensin converting enzyme polymorphisms with salt-sensitive hypertension and early renal injury. Zhonghua Yi Xue Yi Chuan Xue Za Zhi 2008; 25: 132-135.

42 Gong P, Shen G, Peng H, Luo Y, Shen Y, Zhao X. Association of the polymorphisms of sodium transport related genes with essential hypertension. Zhonghua Yi Xue Yi Chuan Xue Za Zhi 2009; 26: 91-94.

43 Lin H, Chen H, Luo J, Wu X, Chen Y, Li D, Chen X. Association among ACE, CYP11B2 and $\alpha$-adducin gene polymorphisms with pulse pressure. Xin Xue Guan Kang Fu Yi Xue Za Zhi 2009; 18: 204-207.

44 Niu WQ, Zhang Y, Ji KD, Gao PJ, Zhu DL. Lack of association between alpha-adducin G460W polymorphism and hypertension: evidence from a case-control study and a meta-analysis. J Hum Hypertens 2010; 24: 467-474.

45 Iwai N, Tamaki S, Nakamura Y, Kinoshita M. Polymorphism of alpha-adducin and hypertension. Lancet 1997; 350: 369.

46 Manunta P, Cusi D, Barlassina C, Righetti M, Lanzani C, D’Amico M, Buzzi L, Citterio L, Stella P, Rivera R, Bianchi G. Alpha-adducin polymorphisms and renal sodium handling in essential hypertensive patients. Kidney Int 1998; 53: 1471-1478.

47 Manunta P, Barlassina C, Bianchi G. Adducin in essential hypertension. FEBS Lett 1998; 430: 41-44.

48 Beeks E, Kessels AG, Kroon AA, van der Klauw MM, de Leeuw PW. Genetic predisposition to salt-sensitivity: a systematic review. J Hypertens 2004; 22: 1243-1249.

49 Glorioso N, Manunta P, Filigheddu F, Troffa C, Stella P, Barlassina C, Lombardi C, Soro A, Dettori F, Parpaglia PP, Alibrandi MT, Cusi D, Bianchi G. The role of alphaadducin polymorphism in blood pressure and sodium handling regulation may not be excluded by a negative association study. Hypertension 1999; 34: 649-654.

50 Barlassina C, Schork NJ, Manunta P, Citterio L, Sciarrone M, Lanella G, Bianchi G, Cusi D. Synergistic effect of alpha-adducin and ACE genes causes blood pressure changes with body sodium and volume expansion. Kidney Int 2000; 57: 1083-1090.

51 Sciarrone MT, Stella P, Barlassina C, Manunta P, Lanzani C, Bianchi G, Cusi D. ACE and alpha-adducin polymorphism as markers of individual response to diuretic therapy. Hypertension 2003; 41: 398-403.

52 Ciechanowicz A, Widecka K, Drozd R, Adler G, Cyryłowski L, Czekalski S. Lack of association between Gly460Trp polymorphism of alpha-adducin gene and salt sensitivity of blood pressure in Polish hypertensives. Kidney Blood Press Res 2001; 24: 201-206.

53 Grant FD, Romero JR, Jeunemaitre X, Hunt SC, Hopkins PN, Hollenberg NH, Williams $\mathrm{GH}$. Low-renin hypertension, altered sodium homeostasis, and an alpha-adducin polymorphism. Hypertension 2002; 39: 191-196.

54 Wang R, Zhong B, Liu Y, Wang C. Association between alpha-adducin gene polymorphism (Gly460Trp) and genetic predisposition to salt sensitivity: a meta-analysis. J App/ Genet 2010; 51: 87-94.

55 Du R, Xiao C, Cavalli-Sforza LL. Genetic distances between Chinese groups calculated on gene frequencies of 38 loci. Sci China C Life Sci 1997; 40: 613-621.

56 Chu JY, Huang W, Kuang SQ, Wang JM, Xu JJ, Chu ZT, Yang ZQ, Lin KQ, Li P, Wu M, Geng ZC, Tan CC, Du RF, Jin L. Genetic relationship of populations in China. Proc Nat' Acad Sci USA 1998; 95: 11763-11768.

57 Shi H, Dong YL, Wen B, Xiao CJ, Underhill PA, Shen PD, Chakraborty R, Jin L, Su B. Y-chromosome evidence of southern origin of the East Asian-specific haplogroup 03-M122. Am J Hum Genet 2005; 77: 408-419.

58 Zhang ZB. An analysis of the physical characteristics of modern Chinese. Ren Lei Xue Xue Bao 1988; 7: 314-323.

59 Zhang ZB. Evidence for origin of Chinese populations: time and space changes of craniometric traits. Di Si Ji Yan Jiu 1999; 2: 113-124.

60 Zhang HG. The distribution of dermatoglyphics parameters in fifty-two Chinese populations. Ren Lei Xue Xue Bao 1988; 7: 39-45.

61 Comas D, Plaza S, Wells RS, Yuldaseva N, Lao O, Calafell F, Bertranpetit J. Admixture, migrations, and dispersals in Central Asia: evidence from maternal DNA lineages. Eur J Hum Genet 2004; 12: 495-504.

62 Yao YG, Kong QP, Wang CY, Zhu CL, Zhang YP. Different Matrilineal Contributions to Genetic Structure of Ethnic Groups in the Silk Road Region in China. Mol Biol Evol 2004; 21: 2265-2280.

63 Cavalli-Sforza LL, Menozzi P, Piazza A. The History and Geography of Human Genes. Princeton University Press: Princeton, 1994.

64 Liu K, Liu J, Huang Y, Liu Y, Lou Y, Wang Z, Zhang H, Yan S, Li Z, Wen S. Alphaadducin Gly460Trp Polymorphism and Hypertension Risk: a Meta-analysis of 22 Studies including 14303 Cases and 15961 Controls. PloS One 2010; 5: e13057.

65 Iwasaki M, Kobayashi K, Suzuki H, Anan K, Ohno S, Geng Z, Li G, Inoko H. Polymorphism of the ABO blood group genes in Han, Kazak and Uygur populations in the Silk Route of northwestern China. Tissue Antigens 2000; 56: 136-142.

66 Li D, Zhang GL, Lou YQ, Li Q, Wang X, Bu XY. Genetic polymorphisms in MDR1 and CYP3A5 and MDR1 haplotype in mainland Chinese Han, Uygur and Kazakh ethnic groups. J Clin Pharm Ther 2007; 32: 89-95.

67 Zhang H, Tao G, Wu Q, Liu J, Gao Y, Chen R, Leng X. Studies on the frequencies of vitamin $D$ receptor gene polymorphism in postmenopausal women of Han and Kazak nationality in China. J Bone Miner Metab 2001; 19: 321-323. 


\section{APPENDIX}

\section{Criteria of methodologic quality assessment for genetic association case/control study on hypertension}

A. Representativeness of cases

Consecutive/randomly selected from case population with clearly defined sampling frame

Consecutive/randomly selected from case population without clearly defined sampling frame or extensive inclusion/exclusion criteria

No method of selection described

2

1

B. Representativeness of controls

Controls were consecutive/randomly drawn from the same sampling frame (ward/community) as cases

Controls were consecutive/randomly drawn from a different sampling frame than cases

No method of selection described

C. Ascertainment of hypertension cases

Clearly described objective criteria for diagnosis of hypertension

Diagnosis of hypertension by patient self-report or by patient history

Not described

D. Ascertainment of controls

Clinical examinations were performed on controls to prove that controls did not have hypertension Article merely stated that controls were subjects who did not report hypertension; no objecting testing Not described

E. Ascertainment of genotyping examination Genotyping done under "blind" conditions

Unblended or not mentioned

F. Test for Hardy-Weinberg equilibrium

Hardy-Weinberg equilibrium in control group Hardy-Weinberg disequilibrium in control group

Hardy-Weinberg equilibrium not checked

G. Association assessment

Assessed association between genotypes and hypertension with appropriate statistics and adjustment for confounders 